\title{
Cache-aided mobile edge computing for B5G wireless communication networks
}

\author{
Junjuan Xia ${ }^{{ }^{*}}$ (D), Chao Li ${ }^{1}$, Xiazhi Lai ${ }^{2}$, Shiwei Lai ${ }^{1}$, Fusheng Zhu ${ }^{3}$, Dan Deng ${ }^{4}$ and Liseng Fan ${ }^{1}$
}

\begin{abstract}
This paper investigates a cache-aided mobile edge computing (MEC) network, where the source offloads the computation task to multiple destinations with computation capacity, with the help of a cache-aided relay. For the proposed cache-aided MEC networks, two destination selection criteria have been proposed to maximize the computation capacity of the selected destination, the channel gain of relay link and the channel gain of direct link, respectively. Similarly, three destination selection criteria have been proposed for the cache-free MEC networks based on the computation capacities of destinations and the channel gains of transmission links, respectively. To evaluate the system performance regarding the latency constraint, we provide the outage probability for the proposed network which is defined based on the transmission-plus-computation time. Our analysis suggests that caching can significantly alleviate the impact of increasing the size of computation task, since only half of the transmission time of cache-free network is required. However, the cache-aided network can not fully exploit the signal from both direct and relay links, thus the improvement by caching is less significant in the high signal-to-noise ratio (SNR) region, compared with the cache-free network employing the destination with maximal channel gain of direct link. Numerical results are given to validate our analysis.
\end{abstract}

Keywords: Cache, MEC, B5G, Outage probability, Relay

\section{Introduction}

Mobile edge computing (MEC) has been emerging as a powerful tool to support real-time and high-quality services, such as virtual reality and tactile internet applications [1]. Due to the limited computation capacity and storage, mobile devices have to offload the computation tasks to computing access points, e.g., cloud datacenters, which possess powerful computing capability and significantly reduce the computation latency. Therefore, it is of vital importance to accommodate traditional wireless networks to support latency-sensitive computation task for the fifth-generation (5G) wireless networks. Accordingly, the authors in [2-4] have proposed the concept of mobile edge computing (MEC) wireless communications networks, where the computation tasks can be offloaded

*Correspondence: xiajunjuan@gzhu.edu.cn

'The School of Computer Science, Guangzhou University, Guangzhou, China ${ }^{3}$ Guangdong New Generation Communication and Network Innovative Institute (GDCNi), Guangzhou, China

${ }^{4}$ Guangzhou Panyu Polytechnic, Guangzhou, China

Full list of author information is available at the end of the article to the edge nodes with computation capacity through the wireless links.

Cooperative relaying is a promising technique to improve the spectrum efficiency, strengthen the system security, and enhance the network connectivity [5-8]. To improve the performance of MEC networks, cooperative relaying has been proposed to improve the transmission rate, which therefore reduces the latency and the energy efficiency [9-11]. Specifically, Cao et al. in [9] proposed to utilize relay nodes consisting of retransmission and cooperative computation to improve the performance of MEC network, and the authors designed an energyefficient algorithms for the proposed network. In [10], Hu et al. investigated the wireless powered cooperation network, where the access terminals are powered by the radio signal transmitted from the MEC base station, and the authors optimized the transmit power for the proposed system. Based on above works, Wen et al. in [11] further considered the full-duplex relaying scenario and jointly optimized the task offloading and computing scheme.

During the peak traffic, the network transmission confronts a huge challenge of congestion, which causes a 
severe issue of latency $[12,13]$. Therefore, caching was proposed to effectively alleviate the congestion of traffic in wireless networks [14-16]. To mitigate the congestion of communications traffic and improve the network performance, the convergency of caching and MEC has become a major topic in the field of wireless communications [17-20]. With the aid of caching, the stressing burden of offloading can be alleviated; thus, the throughput and energy efficiency of MEC networks can be enhanced. In particular, the authors in [9] proposed to jointly optimize the cache placement and computation task offloading. Moreover, Tan et al. in [21] investigated the cache-enabled MEC network and designed a virtual resource allocation scheme for the network with heterogeneous services. Further, Zhou et al. in [22] optimized the cache placement and computation task offloading strategy from an information-centric perspective. Besides the above research, there have been some researches on the newly developed materials [23-26], which can be used in wireless networks for both transmission and improving the environments.

In this paper, we study the cache-aided MEC networks, where the task offloading from the source to the destinations with computation capacities is assisted by a cache-enabled relay. Also, we compare the performance of cache-aided relay network with that of cache-free network. In particular, we propose several destination selection criteria with the purposes of maximizing the computation capacity of destination, the channel gain of direct and relaying links, respectively. As we consider the latency-constraint MEC networks, the outage probabilities based on maximal transmission-pluscomputation time for the proposed criteria are derived under Rayleigh fading channels. Our analysis suggests that caching can significantly alleviate the impact of the increasing size of computation task, as only half of the transmission time of cache-free network is required. However, the cache-aided network can not fully exploit both direct and relaying links, and the enhancement by caching is less significant in the high signal-to-noise ratio (SNR) region. Simulation results are given to validate the analysis.

The key contributions of this paper are summarized as follows:

- We study the cache-aided MEC networks, where the task offloading from the source to the destinations with computation capacities is assisted by a cache-enabled relay.

- Several destination selection criteria are utilized to choose one best destination, in order to enhance the network performance.

- For each selection criterion, we present the analytical outage probability analysis in order to evaluate the network performance in the whole range of SNR.

\section{Methods/experimental}

As depicted in Fig. 1, we consider a MEC network, where the source $S$ aims to offload a computation task with size of $L$ bits to the destination $D_{n}(n \in[1, N])$ with the assistance of a cache-aided relay $R$. Specifically, $D_{n}$ is equipped with a CPU of cycle frequency of $\delta_{n}$, and we assumes that each bit of computation task requires $K \mathrm{CPU}$ cycles to complete the computation. For the sake of convenience, we assume that each node in the considered networks is equipped with a single antenna, and the flat

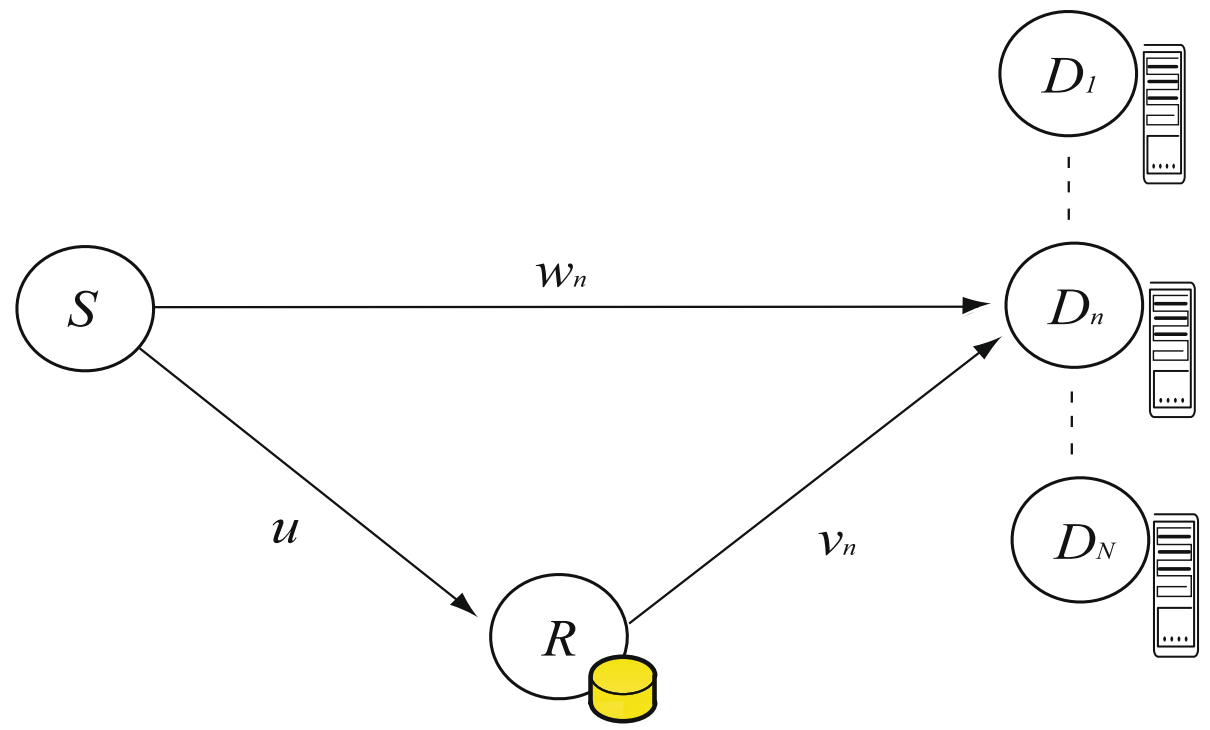

Fig. 1 Cache-aided mobile edge computing networks 
fading Rayleigh channels are considered as well. Based on the latency constraint for transmission and computation time, we define the outage event for the proposed system. To enhance the system performance, we propose two destination selection criteria for the cache-aided network and three destination selection criteria for the cache-free network, aiming to select the destination with maximal computation capacity and maximal channel gain form direct or relay link, respectively.

\section{System model}

For a cache-aided network where the relay is equipped with cache, the computation task can be transmitted from the relay; thus, the source-to-relay and sourceto-destination can be reduced. Therefore, the computation time and transmission time for $D_{n}$ are respectively given by

$$
\begin{aligned}
& t_{C, n}=\frac{K L}{\delta_{n}}, \\
& t_{T, n}=\frac{L}{B \log _{2}\left(1+\frac{P v_{n}}{\sigma^{2}}\right)},
\end{aligned}
$$

where $B$ is the dedicated bandwidth for the transmission of computation task, $v_{n} \sim \mathbf{E}(\beta)$ is the channel gain of the $R$-to- $D_{n}$ link [27-29], $P$ is the transmit power at the source and relay, and $\sigma^{2}$ is the variance of the additive Gaussian noise $n \sim \mathcal{C N}\left(0, \sigma^{2}\right)[30,31]$. Note that we consider a latency-constraint scenario, in which the maximal transmission plus computation time is fixed to $T$, where $T>\frac{K L}{\min _{n \in[1, N]}\left(\delta_{n}\right)}$ is assumed to ensure the implementation of task computing for arbitrary $D_{n}$. Therefore, we adjust the traditional definition of outage event and further define that the outage at destination $D_{n}$ occurs when the required transmission plus computation time is greater than $T$, i.e.,

$$
\frac{K L}{\delta_{n}}+\frac{L}{B \log _{2}\left(1+\frac{P v_{n}}{\sigma^{2}}\right)}>T
$$

which is equivalent to

$$
\frac{P v_{n}}{\sigma^{2}}<\gamma_{t h, n}^{(1)}
$$

where $\gamma_{t h, n}^{(1)}$ denotes the outage threshold for the received $\mathrm{SNR}$ at $D_{n}$ for the cache-aided network, and $\gamma_{t h, n}^{(1)}$ is defined as

$$
\gamma_{t h, n}^{(1)}=2^{\frac{L}{B\left(T-\frac{K L}{\delta}\right)}}-1
$$

For the cache-free network where there is no cache equipped at the relay, two transmission time slots are required. In detail, the source conveys the computation task to both relay and $D_{n}$ during the first phase, and the relay decodes and transmits the computation task to $D_{n}$ during the next phase. The selection combining receiver is employed at the destination to combine the two-branches signal. Accordingly, the computation time and transmission time for $D_{n}$ are respectively given by

$$
\begin{aligned}
& t_{C, n}=\frac{K L}{\delta_{n}}, \\
& t_{T, n}=\frac{2 L}{B \log _{2}\left(1+\frac{P \max \left(w_{n}, \min \left(u, v_{n}\right)\right)}{\sigma^{2}}\right)},
\end{aligned}
$$

where $u \sim \mathbf{E}(\alpha)$ is the channel gain of the $S$-to- $R$ link and $w_{n} \sim \mathbf{E}(\varepsilon)$ is the channel gain of the $S$-to- $D_{n}$ link. Similarly, the outage event occurs when

$$
\frac{P \max \left(w_{n}, \min \left(u, v_{n}\right)\right)}{\sigma^{2}}<\gamma_{t h, n}^{(2)},
$$

where $\gamma_{t h, n}^{(2)}$ is the outage threshold for the received SNR at $D_{n}$ for the cache-aided network, and $\gamma_{t h, n}^{(2)}$ is defined as

$$
\gamma_{t h, n}^{(2)}=2^{\frac{2 L}{B\left(T-\frac{K L}{\delta_{n}}\right)}}-1
$$

\section{Destination selection criterion}

In this section, we aim to design the destination selection 1 criteria to enhance the performance for the cacheaided network and the cache-free network, respectively. Different from the traditional wireless communications networks, MEC wireless networks consider the computation capacities of destinations, in which the traditional selection criteria may not be suitable. Note that the optimal destination selection criterion is very complicated and unrealistic in practice, we thus propose the following suboptimal criteria for the considered networks. The details of the selection criteria are illustrated as follows.

For the cache-aided network, we propose two destination selection criteria, which aim to achieve maximal computation capacity at the destination and maximal channel gain for the relay-to-destination link, respectively. The destination $n^{*}$ is selected based on the following criteria.

- Criterion Cache-Aided-I

$$
n^{*}=\arg \max _{n \in[1, N]}\left(\delta_{n}\right)
$$

- Criterion Cache-Aided-II

$$
n^{*}=\arg \max _{n \in[1, N]}\left(v_{n}\right)
$$

For the cache-free network, we propose three destination selection criteria. The destination $n^{*}$ is chosen according to the following criteria.

- Criterion Cache-free-I

$$
n^{*}=\arg \max _{n \in[1, N]}\left(\delta_{n}\right)
$$

\footnotetext{
${ }^{1}$ Note that in literatures [14], user selection is used instead of destination
} selection. However, both of them represent the same meaning in practice. 
- Criterion Cache-free-II

$$
n^{*}=\arg \max _{n \in[1, N]}\left(v_{n}\right)
$$

- Criterion Cache-free-III

$$
n^{*}=\arg \max _{n \in[1, N]}\left(w_{n}\right)
$$

We see that for criterion Cache-free-I, the destination with maximal computation capacity is selected. For criterion Cache-free-II, the destination with maximal channel gain of relay-to destination link is selected. For criterion Cache-free-III, the destination with maximal channel gain of source-to destination link is selected.

\section{Outage performance analysis}

In this section, the exact outage probabilities for the proposed networks are derived. Moreover, some insights on the system are given to better analyze the proposed system.

\subsection{Cache-aided network}

For the cache-aided network, from criterion CacheAided-I, we see that the destination with maximal computation capacity $\delta_{n^{*}}=\max _{n \in[1, N]}\left(\delta_{n}\right)$ is selected. Therefore, the outage event happens when the $R$-to- $\delta_{n^{*}}$ link cannot support the transmission of computation task, and the outage probability for criterion Cache-Aided-I is given by

$$
P_{\text {out }, \mathrm{I}}^{(1)}=\operatorname{Pr}\left(\frac{P v_{n}^{*}}{\sigma^{2}}<\gamma_{t h, n^{*}}^{(1)}\right),
$$

where $v_{n}^{*}$ can be replaced by $v_{n}$ since the selection of destination does not affect the relay-to-destination link. Using the probability density function (PDF) of random variable (RV) $v_{n}, f_{v_{n}}(x)=\frac{1}{\beta} e^{-\frac{x}{\beta}}$, we can obtain $P_{\text {out }, \mathrm{I}}^{(1)}$ as [32]

$$
\begin{aligned}
P_{\mathrm{out}, \mathrm{I}}^{(1)} & =\int_{0}^{\frac{\gamma_{\text {th n }}^{(1)} \text { * }^{2}}{P}} \frac{1}{\beta} e^{-\frac{x}{\beta}} d x \\
& =1-e^{-\frac{\gamma_{t h, n^{*} \sigma^{2}}^{(1)}}{P \beta}} .
\end{aligned}
$$

From criterion Cache-Aided-II, we see that the destination with maximal channel gain of relay-to-destination link $v_{n^{*}}=\max _{n \in[1, N]}\left(v_{n}\right)$ is selected. Also, the computation capacity of the selected destination varies with identical probability of using computation capacity $\delta_{n}$. Note that the channel gains of $D_{n}$ vary with different time slot and the selected destinations $D_{n}^{*}$ s are of different computation capacities. Thus, the outage threshold for each transmissions varies. However, for criterion CacheAided-II, the probabilities of selecting $D_{n}$ are identical, i.e., $\operatorname{Pr}\left(\gamma_{t h, n^{*}}^{(1)}=\gamma_{t h, n}^{(1)}\right)=1 / N$. Therefore, the outage probability for criterion Cache-Aided-I is given by

$$
\begin{aligned}
P_{\text {out }, \text { II }}^{(1)} & =\operatorname{Pr}\left(\frac{P v_{n}^{*}}{\sigma^{2}}<\gamma_{t h, n^{*}}^{(1)}\right) \\
& =\frac{1}{N} \sum_{n=1}^{N} \operatorname{Pr}\left(\frac{P v_{n}^{*}}{\sigma^{2}}<\gamma_{t h, n}^{(1)}\right) .
\end{aligned}
$$

Using the PDF of RV $v_{n}, f_{v_{n}^{*}}(y)=\frac{1}{\beta} e^{-\frac{y N}{\beta}}$, we can obtain $P_{\text {out,II }}^{(1)}$ as

$$
\begin{aligned}
P_{\text {out }, \text { II }}^{(1)} & =\frac{1}{N} \sum_{n=1}^{N} \int_{0}^{\frac{\gamma_{t h, n}^{(1)} \sigma^{2}}{P}} \frac{1}{\beta} e^{-\frac{y N}{\beta}} d y \\
& =\frac{1}{N} \sum_{n=1}^{N} 1-e^{-\frac{\gamma_{\text {th, }}^{(1)} \sigma^{2} N}{P \beta}} .
\end{aligned}
$$

\subsection{Cache-free network}

For the cache-free network, from criterion Cache-freeI, we see that the destination with maximal computation capacity $\delta_{n^{*}}=\max _{n \in[1, N]}\left(\delta_{n}\right)$ is selected. Therefore, the outage probability for criterion Cache-free-I is given by

$$
\begin{aligned}
P_{\text {out }, \mathrm{I}}^{(2)}= & \operatorname{Pr}\left(\frac{P \max \left(w_{n}^{*}, \min \left(u, v_{n}^{*}\right)\right)}{\sigma^{2}}<\gamma_{t h, n^{*}}^{(2)}\right) \\
\stackrel{(a)}{=} & \operatorname{Pr}\left(\frac{P w_{n}^{*}}{\sigma^{2}}<\gamma_{t h, n^{*}}^{(2)}\right) \\
& \times\left(1-\operatorname{Pr}\left(\frac{P \min \left(u, v_{n}^{*}\right)}{\sigma^{2}}>\gamma_{t h, n^{*}}^{(2)}\right)\right),
\end{aligned}
$$

where step (a) follows the law of total probability. We see that the first term denotes the outage probability of direct link and the second terms denotes the outage probability of relay link, which means that the cache-free relay network can fully exploit signals from both direct and relay branches. However, the cache-aided network can only exploit the relay-branch signal. Further, we can rewrite $P_{\text {out,I }}^{(2)}$ as

$$
\begin{aligned}
P_{\mathrm{out}, \mathrm{I}}^{(2)}= & \left(1-\operatorname{Pr}\left(\frac{P u}{\sigma^{2}}>\gamma_{t h, n^{*}}^{(2)}, \frac{P v_{n}^{*}}{\sigma^{2}}>\gamma_{t h, n^{*}}^{(2)}\right)\right) \\
& \times \operatorname{Pr}\left(\frac{P w_{n}^{*}}{\sigma^{2}}<\gamma_{t h, n^{*}}^{(2)}\right),
\end{aligned}
$$

Similarly, $w_{n}$ and $v_{n}^{*}$ can be replaced by $w_{n}$ and $v_{n}$, respectively. Substituting the PDFs of RV $u, v_{n}$, and $w_{n}$, i.e., $f_{u}(x)=\frac{1}{\alpha} e^{-\frac{x}{\alpha}}, f_{v_{n}}(y)=\frac{1}{\beta} e^{-\frac{y}{\beta}}$, and $f_{w_{n}}(z)=\frac{1}{\varepsilon} e^{-\frac{z}{\varepsilon}}$, we can compute $P_{\text {out,I }}^{(1)}$ as 


$$
\begin{aligned}
P_{\text {out }, \mathrm{I}}^{(2)}= & \left(1-\int_{\frac{\gamma_{t h, n^{*}}^{(2)}}{P}}^{\infty} \frac{1}{\alpha} e^{-\frac{x}{\alpha}} d x \times \int_{\frac{\gamma_{t h, n^{*}}^{(2)}}{P}}^{\infty} \frac{1}{\beta} e^{-\frac{y}{\beta}} d y\right) \\
& \times \int_{0}^{\frac{\gamma_{t h, n^{*} \sigma^{2}}^{(2)}}{P}} \frac{1}{\varepsilon} e^{-\frac{z}{\varepsilon}} d z \\
= & \left(1-e^{-\frac{\gamma_{t h, n^{*}}^{(1)} \sigma^{2}}{P \alpha}}-\frac{\gamma_{t h, n^{*} \sigma^{2}}^{(1)}}{P \beta}\right) \times\left(1-e^{-\frac{\gamma_{t h, n^{*} \sigma^{2}}^{(1)}}{P \varepsilon}}\right) .
\end{aligned}
$$

From criterion Cache-free-II, we see that the destination with maximal channel gain of relay-to-destination link $v_{n^{*}}=\max _{n \in[1, N]}\left(v_{n}\right)$ is selected. However, for criterion Cache-Free-II, the probabilities of selecting $D_{n}$ are identical, i.e., $\operatorname{Pr}\left(\gamma_{t h, n^{*}}^{(2)}=\gamma_{t h, n}^{(2)}\right)=1 / N$. Therefore, the outage probability for criterion Cache-free-II is given by

$$
\begin{aligned}
P_{\text {out,II }}^{(2)}= & \frac{1}{N} \sum_{n=1}^{N} \operatorname{Pr}\left(\frac{P \max \left(w_{n}^{*}, \min \left(u, v_{n}^{*}\right)\right)}{\sigma^{2}}<\gamma_{t h, n}^{(2)}\right) \\
= & \frac{1}{N} \sum_{n=1}^{N}\left(1-\operatorname{Pr}\left(\frac{P u}{\sigma^{2}}>\gamma_{t h, n}^{(2)}, \frac{P v_{n}^{*}}{\sigma^{2}}>\gamma_{t h, n}^{(2)}\right)\right) \\
& \times \operatorname{Pr}\left(\frac{P w_{n}}{\sigma^{2}}<\gamma_{t h, n}^{(2)}\right) .
\end{aligned}
$$

Using the PDFs of RVs $u, v_{n}$ and $w_{n}$, i.e., $f_{u}(x)=\frac{1}{\alpha} e^{-\frac{x}{\alpha}}$, $f_{v_{n}^{*}}(y)=\frac{1}{\beta} e^{-\frac{y N}{\beta}}$ and $f_{w_{n}}(z)=\frac{1}{\varepsilon} e^{-\frac{z}{\varepsilon}}$, we can obtain $P_{\text {out }, \mathrm{II}}^{(2)}$ as

$$
\begin{aligned}
P_{\text {out }, \mathrm{II}}^{(2)}= & \frac{1}{N} \sum_{n=1}^{N}\left(1-\int_{\frac{\gamma_{t h, n} \sigma^{2}}{P}}^{\infty} \frac{1}{\alpha} e^{-\frac{x}{\alpha}} d x \int_{\frac{\gamma_{t h, n} \sigma^{2}}{P}}^{\infty} \frac{1}{\beta} e^{-\frac{y N}{\beta}} d y\right) \\
& \times \int_{0}^{\frac{\gamma_{t h, n}^{(2)} \sigma^{2}}{P}} \frac{1}{\varepsilon} e^{-\frac{z}{\varepsilon}} d z \\
= & \frac{1}{N} \sum_{n=1}^{N}\left(1-e^{-\frac{\gamma_{t h, n}^{(1)} \sigma^{2}}{P \alpha}-\frac{\gamma_{t h, n}^{(1)} \sigma^{2}}{P \beta}}\right)\left(1-e^{-\frac{\gamma_{t h, n}^{(1)} \sigma^{2}}{P \varepsilon}}\right) .
\end{aligned}
$$

From criterion Cache-free-III, we see that the destination with maximal channel gain of relay-to-destination link $w_{n^{*}}=\max _{n \in[1, N]}\left(w_{n}\right)$ is selected. Similarly, for criterion Cache-Free-III, the probabilities of selecting $D_{n}$ are identical, i.e., $\operatorname{Pr}\left(\gamma_{t h, n^{*}}^{(1)}=\gamma_{t h, n}^{(1)}\right)=1 / N$. Therefore, the outage probability for criterion Cache-free-III is given by

$$
\begin{aligned}
P_{\text {out }, \text { III }}^{(2)}= & \frac{1}{N} \sum_{n=1}^{N} \operatorname{Pr}\left(\frac{P \max \left(w_{n}^{*}, \min \left(u, v_{n}^{*}\right)\right)}{\sigma^{2}}<\gamma_{t h, n}^{(2)}\right) \\
= & \frac{1}{N} \sum_{n=1}^{N}\left(1-\operatorname{Pr}\left(\frac{P u}{\sigma^{2}}>\gamma_{t h, n}^{(2)}, \frac{P v_{n}}{\sigma^{2}}>\gamma_{t h, n}^{(2)}\right)\right) \\
& \times \operatorname{Pr}\left(\frac{P w_{n}^{*}}{\sigma^{2}}<\gamma_{t h, n}^{(2)}\right) .
\end{aligned}
$$

Applying the PDFs of RVs $u, v_{n}$, and $w_{n}$, i.e., $f_{u}(x)=$ $\frac{1}{\alpha} e^{-\frac{x}{\alpha}}, f_{v_{n}^{*}}(y)=\frac{1}{\beta} e^{-\frac{y}{\beta}}$, and $f_{w_{n}}(z)=\frac{1}{\varepsilon} e^{-\frac{z N}{\varepsilon}}$, we can obtain $P_{\text {out }, \text { III }}^{(2)}$ as

$$
\begin{aligned}
P_{\text {out,III }}^{(2)}= & \frac{1}{N} \sum_{n=1}^{N}\left(1-\int_{\frac{\gamma_{t h, n}^{(2)} \sigma^{2}}{P}}^{\infty} \frac{1}{\alpha} e^{-\frac{x}{\alpha}} d x \int_{\frac{\gamma_{t h, n}^{(2)} \sigma^{2}}{P}}^{\infty} \frac{1}{\beta} e^{-\frac{y}{\beta}} d y\right) \\
& \times \int_{0}^{\frac{\gamma_{t h, n}^{(2)} \sigma^{2}}{P}} \frac{1}{\varepsilon} e^{-\frac{z N}{\varepsilon}} d z \\
= & \frac{1}{N} \sum_{n=1}^{N}\left(1-e^{-\frac{\gamma_{t h, n} \sigma^{2}}{P \alpha}-\frac{\gamma_{t h, n}^{(1)} \sigma^{2}}{P \beta}}\right)\left(1-e^{-\frac{\gamma_{t h, n}^{(1)} \sigma^{2} N}{P \varepsilon}}\right) .
\end{aligned}
$$

From the above analysis on the outage performance, we can draw the following insights on the proposed networks.

Remarks 1 From (15), we see that for criteria CacheAided-I and Cache-Free-I, the increase of the number of destination $N$ cannot guarantee the improvement of the network, unless the added destination is of higher computation capacity. This is is because the destination with highest computation capacity is selected, which leads to the loss of diversity on transmission channel.

Remarks 2 From (16)-(18) and (23)-(30), we see that the increase of the number of destination $N$ may results in performance loss of network for criteria Cache-Aided-II, Cache-Free-II and Cache-Free-III. This is due to the fact that the overall outage probability is obtained by averaging the outage probabilities of each destinations with different computation capacities. The involvement of destination with low computation capacity causes the degradation of outage performance.

Remarks 3 Cache-aided networks can effectively improve the system performance by reducing half of the transmission time than the cache-free network. Therefore, criteria Cache-Aided-I and Cache-Aided-II outperform the criteria Cache-Free-I and Cache-Free-II, respectively, in the low SNR region.

Remarks 4 From the outage results, we see that criterion Cache-Free-III can achieve full diversity by exploiting both 
relay and direct links. However, criterion Cache-Aided-II can only exploiting the multi-destination diversity. Therefore, criterion Cache-Free-III outperform criterion CacheAided-II in the high SNR region.

\section{Results and discussion}

In this section, we give the numerical and simulation results regarding the proposed criteria Cache-Aided-I, Cache-Aided-II, Cache-Free-I, Cache-Free-II and CacheFree-III, denoted as "CA-I," "CA-II," "CF-I," "CF-II," and "CF-III," respectively, for convenience sake. Also, we assume the considered nodes are each equipped with one antenna since the limitation of size. The path-loss model is adopted, and we assume the average channel gain of $S$-to- $R$ link $\alpha=8$, the average channel gain of $R$-to- $D_{n}$ link $\beta=5$ and the average channel gain of $S$-to- $D_{n}$ link $\varepsilon=1$. If not specified, we set the number of destination $N=2$, the size of computation task $L=50$ Mbits, the allocated bandwidth $B=100 \mathrm{MHz}$, the transmission plus computation latency threshold $T=0.5 \mathrm{~s}$, transmit SNR $P / \sigma^{2}=15 \mathrm{~dB}$ and $K=10$ [33].

Figure 2 illustrates how outage varies with transmit SNR $P / \sigma^{2}$, where $P / \sigma^{2}$ changes from 0 to $30 \mathrm{~dB}$, when $N=2$, $L=50 \mathrm{Mbits}, K=10, B=100 \mathrm{MHz}$ and $T=0.5 \mathrm{~s}$. From Fig. 2, we see that the simulation outage probabilities match the analytical outage probabilities, which confirms our analysis. Moreover, we see that in the low SNR region, the cache-aided relay network can achieve better performance as cache-aided relay network requires half of the transmission time than cache-free relay network. However, in the high SNR region, for the criteria with the same destination selection purpose, e.g, CA-I and CF-I, or CA-II and CF-II, the criterion for the cache-free relay network outperforms the criterion for cache-aided relay network. This is due to the fact that the cache-free network can exploit one more branch signal than cache-aided relay network, including both the direct and relaying links. However, for the cache-aided relay network, the maximal diversity order is equal to $N$ and no direct-link signal can be exploited.

Figure 3 shows the variations of outage probability with latency threshold $T$, where the values $T$ falls in the range of $[0.1,1]$, when $N=2, L=50$ Mbits, $K=10, B=100$ $\mathrm{MHz}$ and $P / \sigma^{2}=15 \mathrm{~dB}$. From Fig. 3, we confirm the correctness of our analysis by comparing the simulation and analytical outage probabilities. Also, we see that for criteria CA-I and CF-I, the network cannot exploit the diversity of transmission channel, thus the improvement of these two criteria is limited. Moreover, we see that, with the increase of $T$, the outage probabilities for all criteria decrease. This is because for larger value of $T$, the computation task is can be easier to achieve. Also, Eq. (4) suggests that when $T$ is large enough, the outage threshold can barely increase, thus decrease of outage probability with the increase of $T$ decelerates.

Figure 5 depicts how the outage probability changes with different numbers of destinations $N$ when $L=$ 50 Mbits, $K=10, B=100 \mathrm{MHz}, P / \sigma^{2}=15 \mathrm{~dB}$ and $T=0.5 \mathrm{~s}$. Specifically, $N$ varies from 1 to 8 , and the capacity of destination $D_{n}$, i.e., $\delta_{n}$, is equal to $(11-n) \mathrm{GHz}$, in a descending order. This is reasonable since the destination with higher computation capacity is considered first

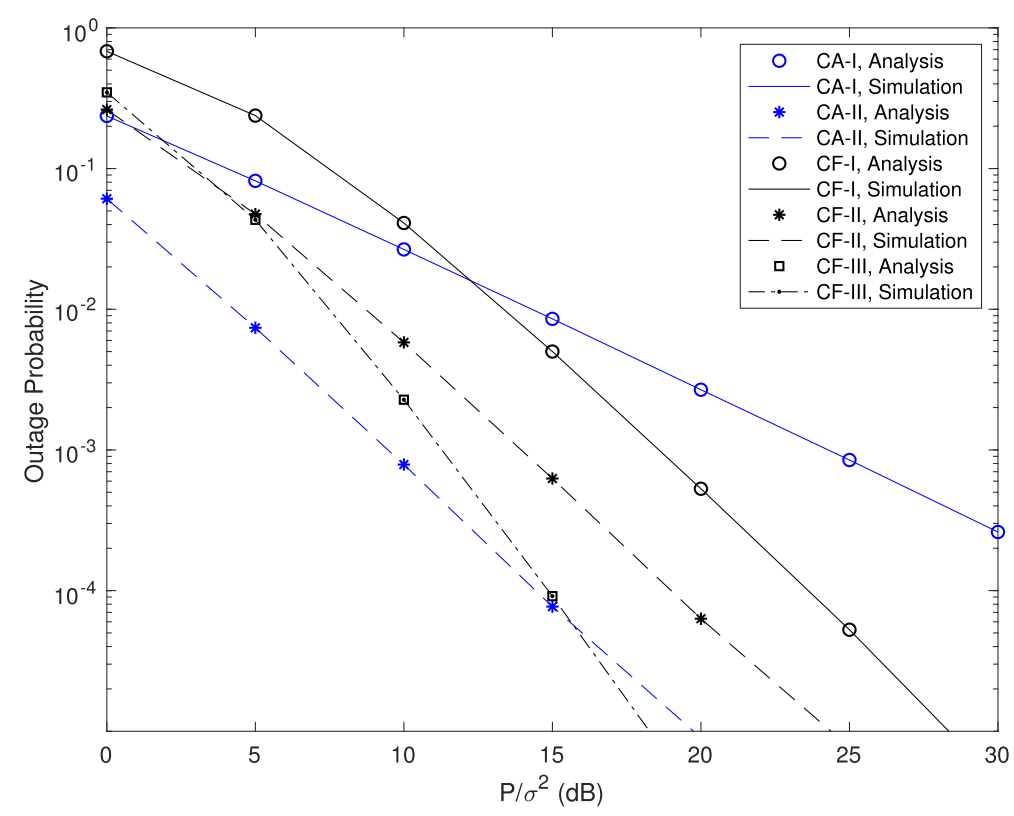

Fig. 2 Outage probability versus $P / \sigma^{2}$ when $N=2, L=50$ Mbits, $K=10, B=100 \mathrm{MHz}$ and $T=0.5 \mathrm{~s}$ 


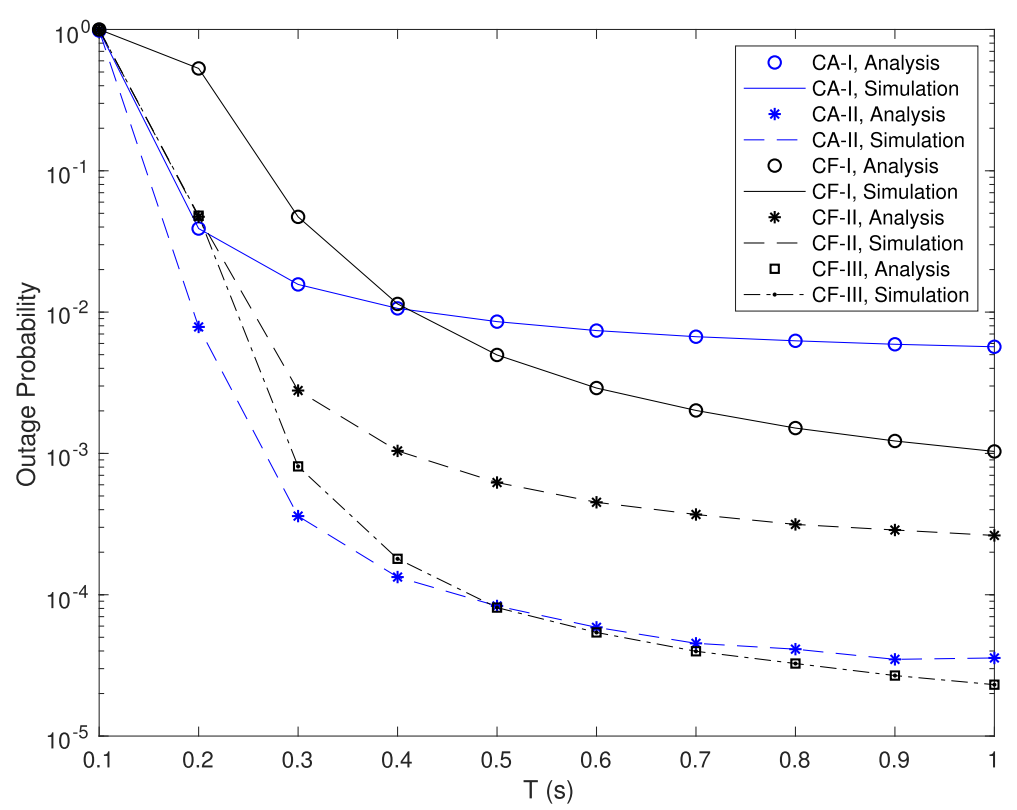

Fig. 3 Outage probability versus $T$ when $N=2, L=50 \mathrm{Mbits}, K=10, B=100 \mathrm{MHz}$ and $P / \sigma^{2}=15 \mathrm{~dB}$

to achieve lower latency (Fig. 4). From Fig. 5, we see that for criteria CA-I and CF-I, the outage probabilities remain the same, since the $\delta_{1}=\max _{n \in[1, N]} \delta_{n}$ and $D_{1}$ is always selected. However, for criterion CF-II, the outage probability increases when $N$ is large. This is due to the fact that the diversity order for CF-II is two and the augment of destination can only improve the second hop of relaying link. Moreover, we assume the later included destinations are of weaker computation capacity, which might cause higher outage probability; thus, from Eq. (30), the overall outage probabilities increase. However, for criteria CA-II and CF-III, the increase of $N$ significantly improves the network performance; thus, the difference of computation capacity of destination can be neglected.

Figure 4 illustrates the effect of the size of computation $\operatorname{task} L$ on the outage performance of system. In specific, $L$ varies from 10 to $100 \mathrm{Mbits}$, and we set $N=2, K=10$, $B=100 \mathrm{MHz}, P / \sigma^{2}=15 \mathrm{~dB}$ and $T=0.5 \mathrm{~s}$. From Fig. 4 ,

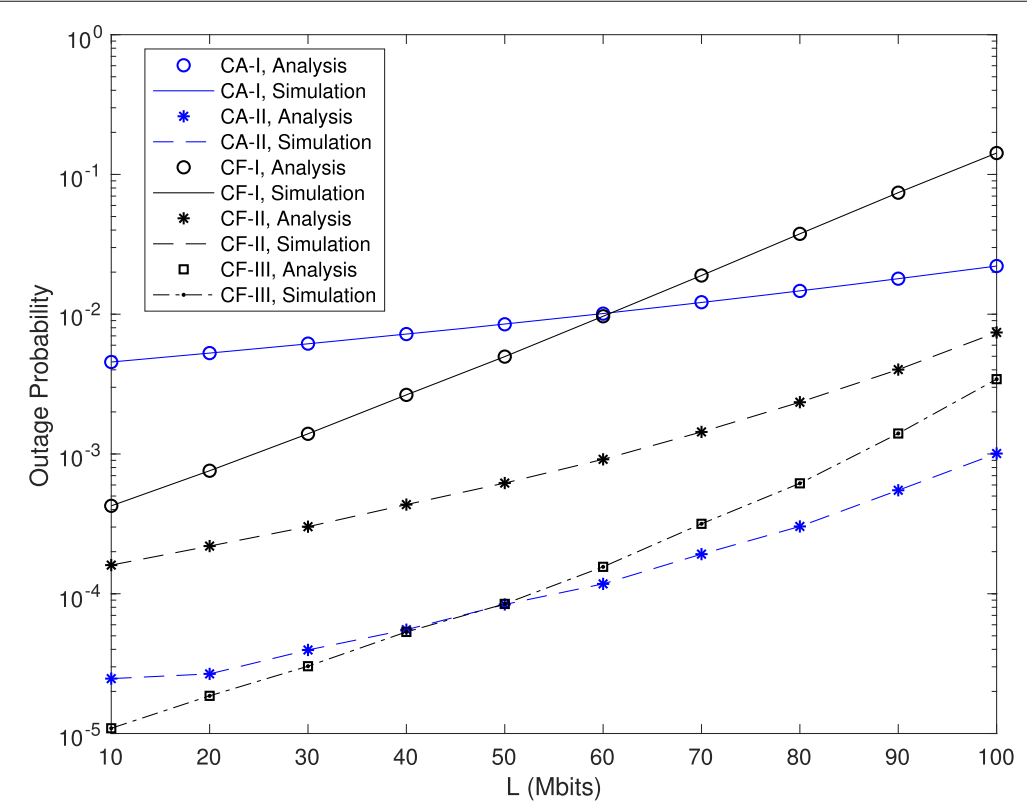

Fig. 4 Outage probability versus $L$ when $N=2, K=10, B=100 \mathrm{MHz}, P / \sigma^{2}=15 \mathrm{~dB}$ and $T=0.5 \mathrm{~s}$ 


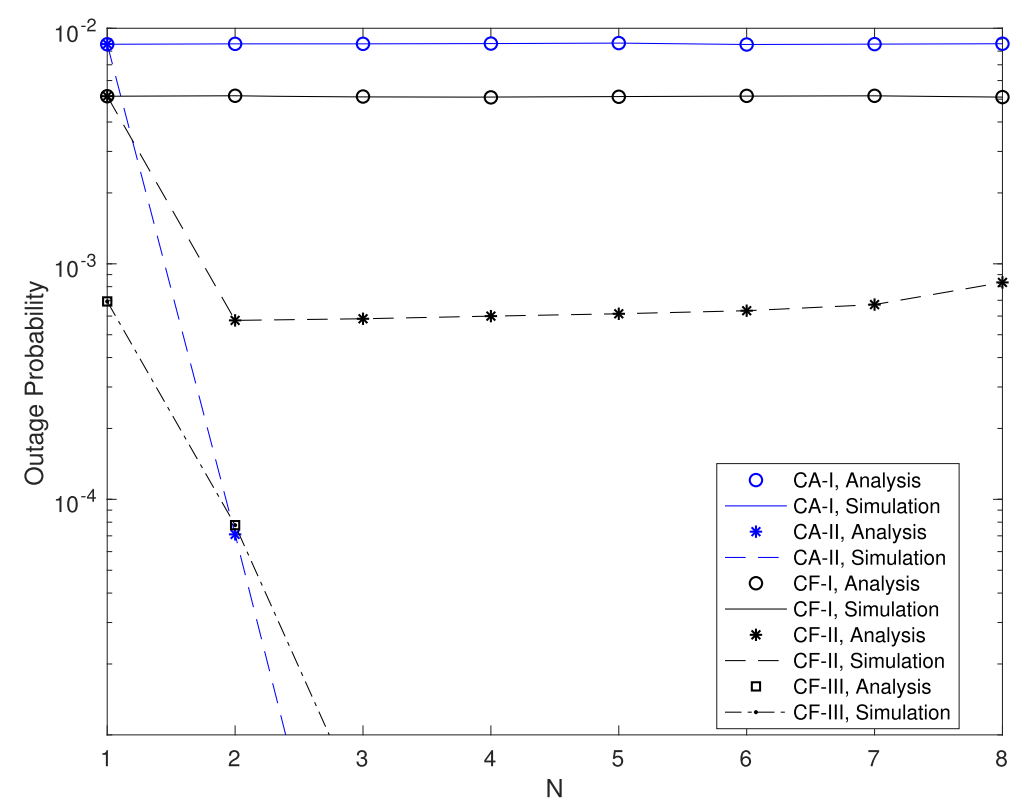

Fig. 5 Outage probability versus $N$ when $L=50$ Mbits, $K=10, B=100 \mathrm{MHz}, P / \sigma^{2}=15 \mathrm{~dB}$ and $T=0.5 \mathrm{~s}$

we see that the increase of $L$ greatly increases the outage probability, since the burdens of transmission is proportional to the size of computation task. Also, the results shows that with the increase of $L$, the cache-free relay networks are more vulnerable than cache-aided networks, since the increase of $L$ doubles the burdens of two-phase transmission for cache-free networks. As a result, with small values of $L$, CF-I outperforms CA-I, yet different phenomenon occurs when the values of $L$ is large.
Figure 6 demonstrates how the outage probability changes with various values of dedicated bandwidth $B$, when $N=2, L=50$ Mbits, $K=10, P / \sigma^{2}=15 \mathrm{~dB}$, and $T=0.5 \mathrm{~s}$. Additionally, $B$ varies from 10 to $100 \mathrm{MHz}$. Also, we see that for criteria CA-I and CF-I, the network only exploits the diversity of computation capacity of destination, which barely affects the network performance as the computation capacity of destination is fixed. Thus, the improvement of these two criteria is not significant.

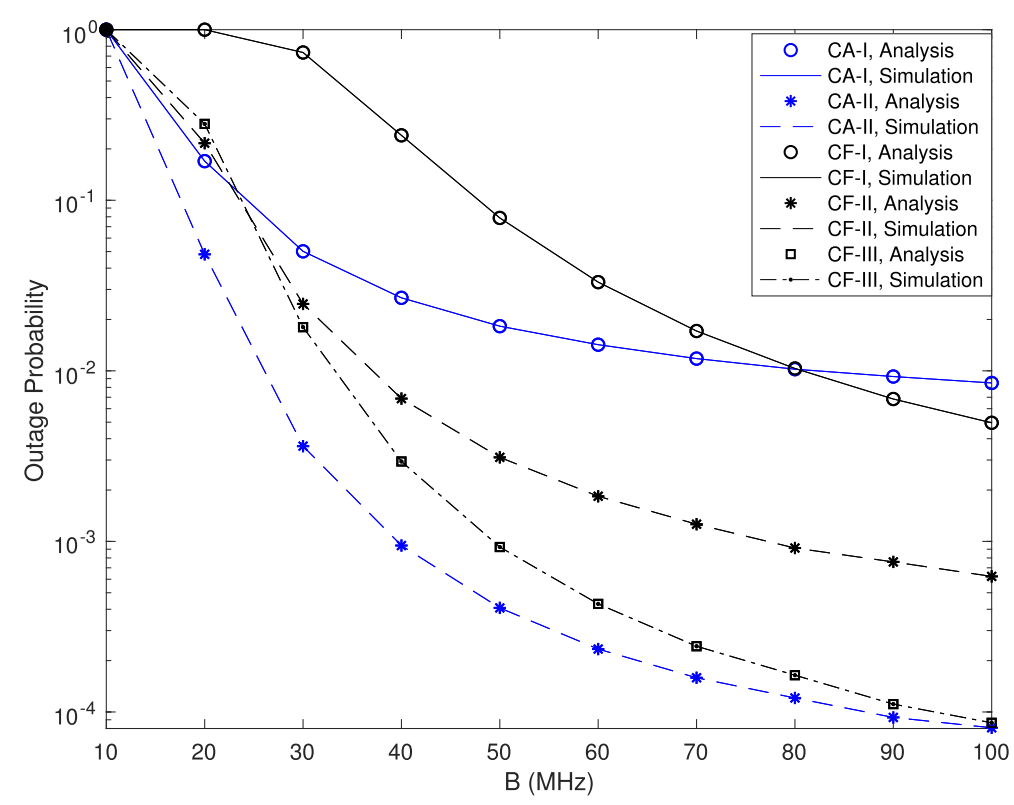

Fig. 6 Outage probability versus $B$ when $N=2, L=50$ Mbits, $K=10, P / \sigma^{2}=15 \mathrm{~dB}$ and $T=0.5 \mathrm{~s}$ 
Moreover, we see that, with the increase of $B$, the outage probabilities for all criteria decrease. This is because for larger value of $B$, the computation task can be easier to achieve. Furthermore, from Eq. (4), we see that when $B$ is large enough, the outage threshold tends to a fixed values, thus decrease of outage probability with the increase of $B$ decelerates.

\section{Conclusions}

This paper studied the cache-aided MEC networks, for which we proposed five destination selection criteria. To evaluate the effectiveness of the proposed criteria, the outage probabilities based on the transmission plus computation time for the propose criteria have been derived. Our results show that the proposed criteria aiming to maximize the channel gain of direct or relaying links can significantly improve the system performance. However, the criteria with purpose of maximizing the computation capacity of destination enjoys very limited benefits of signal diversity. Moreover, analysis suggests that caching can significantly alleviate the impact of increasing the size of computation task as only half of the transmission time of cache-free network is required. Numerical results have been given to validate our analysis. In future works, we will consider the application of this work for IoT networks, such as urban environment improvement [34-36] and environmental monitoring [37-40]. Moreover, we will incorporate the intelligent algorithms such as learning-based algorithms [41, 42], deep learning $[43,44]$, and reinforcement learning [45-47] into the considered system, in order to further enhance the network performance.

\section{Abbreviations \\ CA-I: Cache-Aided-I; CA-II: Cache-Aided-II; CF-I : Cache-Free-I; CF-II: Cache-Free-II; CF-III: Cache-Free-III; MEC: Mobile edge computing; PDF: Probability density function; RV: Random variable; SNR: Signal-to-noise ratio}

\section{Acknowledgements}

This work was supported in part by the NSFC under grant 61871139, in part by the Innovation Team Project of Guangdong Province University under grant 2016KCXTD017, and in part by the Science and Technology Program of Guangzhou under grant 201807010103.

\section{Authors' contributions}

JX gave the main ideas in this work, LC made the simulation experiments, XL derived the formulas, and SL helped write the main manuscript of this work. FZ has helped check the latest reference, re-written the part of the introduction, and provided some insights from the work in this paper. DD has helped improve the language of this manuscript, corrected the grammar errors and clarified some unclear sentences in the manuscript. LF has helped improve the presentation of figures style in this work and helped enhance the novelty of this paper. All authors have read and approved the final manuscript.

\section{Funding}

This work is supported by the National Natural Science Foundation of China (no. 61871139), by the Natural Science Foundation of Guangdong Province (no. 2018A030313736), by the Scientific Research Project of Education Department of Guangdong, China (no. 2017GKTSCX045), by the Science and Technology Program of Guangzhou, China (no. 201707010389), and by the Project of Technology Development Foundation of Guangdong (no. 706049150203).

\section{Availability of data and materials}

The authors state the data availability in this manuscript through the email to the corresponding author.

\section{Competing interests}

The authors declare that they have no competing interests.

\section{Author details \\ ${ }^{1}$ The School of Computer Science, Guangzhou University, Guangzhou, China. ${ }^{2}$ The School of Electronics and Information Technology, Sun Yat-Sen University, Guangzhou, China. ${ }^{3}$ Guangdong New Generation Communication and Network Innovative Institute (GDCNi), Guangzhou, China. ${ }^{4}$ Guangzhou Panyu Polytechnic, Guangzhou, China.}

Received: 29 September 2019 Accepted: 29 November 2019

Published online: 13 January 2020

\section{References}

1. Z. Zhao, A novel framework of three-hierarchical offloading optimization for mec in industrial loT networks. IEEE Trans. Ind. Informa. PP(99), 1-12 (2019)

2. J. Zhao, T. Yang, Y. Gong, et al., Power control algorithm of cognitive radio based on non-cooperative game theory. China Commun. 10(11), 143-154 (2013)

3. J. Zhao, Power Allocation Based on Genetic Simulated Annealing Algorithm in Cognitive Radio Networks. Chin J Electron. 22(1), 177-180 (2013)

4. J. Zha o, Multiband Cooperation for 5 G HetNets: A Promising Network Paradigm. IEEE Veh. Technol. Mag. PP(99), 1-10 (2019). https://doi.org/10. 1109/MVT.2019.2935793

5. L. Fan, N. Zhao, X. Lei, Q. Chen, N. Yang, GK. Karagiannidis, Outage probability and optimal cache placement for multiple amplify-and-forward relay networks. IEEE Trans. Veh. Technol. 67(12), 12373-12378 (2018)

6. X. Lai, W. Zou, et al., DF relaying networks with randomly distributed interferers. IEEE Access. 5, 18909-18917 (2017)

7. J. Yang, D. Ruan, J. Huang, X. Kang, Y.-Q. Shi, An embedding cost learning framework using gan. IEEE Trans. Inf. Forensic. Sec. PP(99), 1-10 (2019)

8. X. Lai, L. Fan, et al., Distributed secure switch-and-stay combining over correlated fading channels. IEEE Trans. Inf. Forensic. Sec. 14(8), 2088-2101 (2019)

9. X. Cao, F. Wang, J. Xu, R. Zhang, S. Cui, Joint computation and communication cooperation for energy-efficient mobile edge computing. IEEE Internet Things J. 6(3), 4188-4200 (2019)

10. X. Hu, K. Wong, K. Yang, Wireless powered cooperation-assisted mobile edge computing. IEEE Trans. Wirel. Commun. 17(4), 2375-2388 (2018)

11. Z. Wen, K. Yang, X. Liu, S. Li, J. Zou, Joint offloading and computing design in wireless powered mobile-edge computing systems with full-duplex relaying. IEEE Access. 6, 72786-72795 (2018)

12. X. Lin, MARL-based distributed cache placement for wireless networks. IEEE Access. PP(99), 1-10 (2019)

13. C. Li, W. Zhou, Enhanced secure transmission against intelligent attacks IEEE Access. 7, 53596-53602 (2019)

14. F. Shi, Secure probabilistic caching in random multi-user multi-UAV relay networks. Phys. Commun. 32, 31-40 (2019)

15. C. Li, Cache-enabled physical-layer secure game against smart UAV-assisted attacks in B5G noma networks. EURASIP J. Wirel. Commun. Netw. PP(99), 1-5 (2019)

16. X. Lin, Probabilistic caching placement in UAV-assisted heterogeneous wireless networks. Phys. Commun. 33, 54-61 (2019)

17. J. Xia, Secure cache-aided multi-relay networks in the presence of multiple eavesdroppers. IEEE Trans. Commun. PP(99), 1-10 (2019)

18. J. Xia, When distributed switch-and-stay combining meets buffer in loT relaying networks. Phys. Commun. PP, 1-9 (2019)

19. S. Lai, Intelligent secure communication for cognitive networks with multiple primary transmit power. IEEE Access. PP(99), 1-7 (2019)

20. Z. Zhao, Intelligent offloading strategy design for relaying mobile edge computing networks. IEEE Access. PP(99), 1-7 (2019)

21. Z. Tan, F. R. Yu, X. Li, H. Ji, V. C. M. Leung, Virtual resource allocation for heterogeneous services in full duplex-enabled SCNs with mobile edge computing and caching. IEEE Trans. Veh. Technol. 67(2), 1794-1808 (2018) 
22. Y. Zhou, F. R. Yu, J. Chen, Y. Kuo, Resource allocation for information-centric virtualized heterogeneous networks with in-network caching and mobile edge computing. IEEE Trans. Veh. Technol. 66(12), 11339-11351 (2017)

23. S. Pan, Synthesis of naked plasmonic/magnetic au/fe3o4 nanostructures by plasmon-driven anti-replacement reaction. Nanotechnology. 30, 1-7 (2019)

24. S. Pan, Plasmon-engineered anti-replacement synthesis of naked cu nanoclusters with ultrahigh electrocatalytic activity. J. Mater. Chem. A. 6 , 18687-18693 (2018)

25. Y. K. Wang, The superior thermal stability and tensile properties of hot rolled W-HfC alloys. Int. J. Refract. Met. Hard Mater. 81, 42-48 (2019)

26. M. M. Wang, Grain size effects of tungsten powder on the micro-structure and mechanical properties of tungsten-based alloys. Mater. Sci. Eng. A. 754, 216-223 (2019)

27. B. Wang, F. Gao, S. Jin, H. Lin, G. Y. Li, Spatial- and frequency-wideband effects in millimeter-wave massive MIMO systems. IEEE Trans. Sig. Process. 66(13), 3393-3406 (2018)

28. H. Xie, F. Gao, S. Zhang, S. Jin, A unified transmission strategy for TDD/FDD massive MIMO systems with spatial basis expansion model. IEEE Trans. Veh. Technol. 66(4), 3170-3184 (2017)

29. X. Hu, C. Zhong, X. Chen, W. Xu, Z. Zhang, Cluster grouping and power control for angle-domain mmwave mimo noma systems. IEEE J. Sel. Top. Sig. Process. 13(5), 1167-1180 (2019)

30. J. Zhao, Q. Li, Y. Gong, K. Zhang, Computation offloading and resource allocation for cloud assisted mobile edge computing in vehicular networks. IEEE Trans. Veh. Technol. 68(8), 7944-7956 (2019)

31. Shanjin Ni, Enhancing Downlink Transmission in MIMO HetNet With Wireless Backhaul. IEEE Trans. Veh. Technol. 68(7), 6817-6832 (2019)

32. I. S. Gradshteyn, I. M. Ryzhik, Table of Integrals, Series, and Products, 7th ed. (Academic, San Diego, 2007)

33. M. K. Simon, M. S. Alouini, Digital Communication over Fading Channels, 2nded. (Wiley, 2005)

34. J. Yang, Inverse optimization of building thermal resistance and capacitance for minimizing air conditioning loads. Renew. Energy. PP, $1-10(2020)$

35. J. Yang, Numerical and experimental study on the thermal performance of aerogel insulating panels for building energy efficiency. Renew. Energy. 138, 445-457 (2019)

36. H. Huang, Optimum insulation thicknesses and energy conservation of building thermal insulation materials in chinese zone of humid subtropical climate. Sust. Cities Soc. 52, 101840 (2020)

37. P. Li, Preparation and optimization of ultra-light and thermal insulative aerogel foam concrete. Const. Build. Mater. 205, 529-542 (2019)

38. J. Yang, Engineering surface and optical properties of TiO2-coated electrospun pvdf nanofibers via controllable self-assembly. Nanomaterials. 8, 1-17 (2018)

39. L. Yang, Preparation and characterization of clay aerogel composites reinforced by calcium sulfate whisker. J. Nanosci. Nanotechnol. 18, 7896-7901 (2018)

40. J. Yang, Modeling and coupling effect evaluation of thermal conductivity of ternary opacifier/fiber/aerogel composites for super-thermal insulation. Mater. Design. 133, 224-236 (2017)

41. C. Fan, Cooling load prediction and optimal operation of HVAC systems using a multiple nonlinear regression model. Energy Build. 197, 7-17 (2019)

42. C. Fan, Analysis of hourly cooling load prediction accuracy with data-mining approaches on different training time scales. Sustain. Cities Soc. 51, 101717 (2019)

43. K. He, A MIMO detector with deep learning in the presence of correlated interference. IEEE Trans. Veh. Technol. PP(99), 1-5 (2019)

44. G. Liu, Deep learning based channel prediction for edge computing networks towards intelligent connected vehicles. IEEE Access. 7, 114487-114495 (2019)

45. C. Li, Y. Xu, Protecting secure communication under UAV smart attack with imperfect channel estimation. IEEE Access. 6(1), 76395-76401 (2018)

46. J. Xia, Intelligent secure communication for internet of things with statistical channel state information of attacker. IEEE Access. PP(99), 1-7 (2019)

47. Y. Xu, Q-learning based physical-layer secure game against multi-agent attacks. IEEE Access. 7, 49212-49222 (2019)

\section{Publisher's Note}

Springer Nature remains neutral with regard to jurisdictional claims in published maps and institutional affiliations.

\section{Submit your manuscript to a SpringerOpen ${ }^{\odot}$ journal and benefit from:}

- Convenient online submission

- Rigorous peer review

- Open access: articles freely available online

- High visibility within the field

- Retaining the copyright to your article

Submit your next manuscript at $>$ springeropen.com 